\title{
Research on Multipath Data Transmission Protocol Based on Wireless Sensor Networks
}

\author{
Guo Weiwei \\ Heilongjiang University of Technology, Jixi 158100, China, \\ gwwguoweiwei@163.com
}

\begin{abstract}
The issue of data transmission in wireless sensor networks was studied. The video data collection was chosen as the object of the study, and the quality of service (QoS) problem of video transmission was analyzed. Video applications were generally characterized by large data size and many QoS requirements, which made the transmission of video always a hard problem. In this dissertation, a cross-layer and multi-path video transmission strategy (MVTS) was presented. MVTS introduced the idea of differentiated service and multipath routing, and operated in both application layer and network layer. In application layer, different types of video frames were distinguished and marked with different tags. Then in network layer these frames were identified by the tags and MVTS forwarded them in different paths. In this way, the key frames could be sent to the sink node reliably. Simulation tests were carried out, and simulation results showed that MVTS extended the network lifetime greatly and provided better assurance for quality of video applications.
\end{abstract}

Keywords: Wireless Sensor Networks, Quality of Service, Data Transmission

\section{Introduction}

Data collection is the most basic task for wireless sensor network. When required data are acquired, nodes deliver them to the sink node via various means and tools [1-2]. Preliminary wireless sensor network collects only simple scalar data like temperature, humidity, concentration, pressure etc. When data are being collected, nodes need only to submit perception data to the converging node, no needs of other services. With increase requirements for monitoring, people become eager to take in more abundant information data such as image, video to the collection scope of wireless sensor network, posing new problems. Many applications have service quality limits in many aspects, including bandwidth, time delay, packet loss rate, distance, jitter ratio, image quality and so on [34]. In such applications, in addition to submit data, data transmission process must accord to relative requirement and norms, and meet certain QoS index. Hence from the perspective of transmission quality guarantee, we discuss data transmission in wireless sensor network [5-7].

We focus mainly on collection and transmission of video data. Video service is a very promising service in the wireless sensor network. It has affluent quantity of information, which enables users to perceive intuitively the site situations of target area and object. Video service can improve the monitoring quality and level of network, realize detection of high accuracy and fine grain in the external circumstances, and implement target recognition, target tracking and video monitoring. Prospective applications will be found in fields like intelligent transportation, tele-medicine, mobile nursing and field investigation. Thus to deal well with transmission of video data will have excellent theoretical significances and promotional values [8-9]. 
In wireless sensor network, there will have following difficulties when video nodes are submitting video data:

(1) Video service has big data volume, of which the transmission requires a great deal of node energy: for sensor nodes which are powered up by batteries, it's a serious challenge about how to complete the video data transmission in the case of limited power supply;

(2) Video service has many requirements for QoS, including bandwidth, time delay, packet loss rate, image quality etc; if they can't be met, it become meaningless even though videos are passed over; wireless sensor network is one wireless multihop transmission network; it has inadequate communication bandwidth and limited node storage space and computational ability; so the key to whether video service can be successfully implemented is whether stable video transmission service can be provided and certain QoS is ensured.

At present in wireless sensor network, no professional solution is developed for an acquisition of video data [10-11]. The general thinking is to find a path between source nodes and destination nodes and send out video data along the path. Shortcomings are found in that way: (1) Nodes on the path will die easily for exhausted energy;

(3) The discovered path is not able to provide favorable QoS support.

To address two problems above, researchers proposed respectively multi-path routing and QoS routing method. The main idea of multi-path routing is using multi-path to spread video data and nodes on multi-path are assigned to resend services. A representative multi-path routing protocol is TPGF, MPMRS, ReInForM, MMSPEED, and MOPC. So far the point of studies on that method is how to find multiple paths; however, very few about how to allocate video data to multiple paths. QoS routing is designed mainly to compute one path which suffices service requirements. The typical method is SPEED, GPSR, REAR, EE-SPEED, REAR, LEAR, and SHPER. Researches on that kind of routing protocol involve some ideas of differentiated service, i.e. for different services, calculating different routes; but for bigger video data, single path is not enough [12].

We introduce here the video data collection in the wireless sensor network and present one multi-path video transmission strategy, i.e. MVTS. MVTS collaborates in network layer and application layer. Also, it combines merits of differentiated service and multipath routing. MVTS differentiates frames of video files in the application layer and with different tags. Such frames after tagging enter to the network layer. Routing modules effect the transmission through unlike paths according to different frames to make sure key frames will reach the converging node safely. Experiments indicate that MVTS can prolong effectively network life cycle and ensure good service quality of video service.

\section{Related Model and Hypothesis}

MVTS makes hypotheses regarding wireless sensor network and video service:

(1) Three types of nodes in the network: video nodes, general nodes and convergence nodes: general nodes are responsible for data transmission service, with lower energy level; video nodes provide data transmission and video collection, with higher energy level than general nodes; convergence nodes take charge of acceptance of video data, with the highest energy level, which thus can be thought infinitely great;

(2) Nodes in the network are randomly deployed, so the same with sink nodes; general nodes are densely deployed so that several communication paths can be found between source nodes and convergence nodes;

(3) Nodes don't have movement ability; after deployment, their positions are 
unchangeable; nodes' communication range is a circular region whose radius $\mathrm{R}$ is the communication distance;

(4) Nodes can get own positional information by means of certain hardware or software technology; that can be implemented by GPS device or positioning algorithm; here we won't discuss how to get coordinates, instead, we assume nodes have known such information;

(5) Video data are encoded and decoded standard MPEG-4 standard. MPEG-4 is a common encoding approach, which is based on target and became international standard in 2000. It realized higher compression efficiency and strong interaction.

\section{Overall Thinking and Structure}

To finish the transmission of video data, MVTS adopts design ideas on multi-path routing, cross-layer design and differentiated service.

\subsection{Multi-Path Routing}

MVTS protocol uses multiple instead of one path to transmit video data. In such a way, data transmission energy consumption is shared equally by nodes on multiple paths to avoid premature dissipation of energy on a single path; on the other hand, multi-path routing can reduce network conflicts, increase throughput and improve network performance in many respects.

\subsection{Cross-Layer Design}

MVTS protocol's design includes network layer and application layer. In the application, it encodes and discerns data; in the network layer, it transmits data. On both layers, it realizes joint design and optimization of data transmission.

\subsection{The Idea of Differentiated Service}

The idea of differentiated service is to provide different service to different business: high-level service for important business and low-level guarantee for insignificant business, which on the whole improves service quality of the network. MVTS expanded that idea and applied on different data in the same service: important data use highly reliable links for higher transmission guarantee; less important data are provided with lower guarantee. Limited quality network resources are preferentially used by significant data, ensuring the transmission QoS of video data in its entirety.

Based on the above idea, we designed MVTS approach. MVTS fulfills the transmission of video data with the collaboration between the application layer and network layer. At source nodes, application layer pack original video data to different image frames and classify them according to the degree of importance. In network layer of source nodes, routing protocol modules choose as per the level of frame different paths through which to send out those frames; after they reach converging node, application layer of the node performs encoding operation of collected data frames to restore the original video data. In the process, since frames of important data receive good service guarantee and they can reach the convergence node at higher possibility, so videos after decoding are of higher quality.

\section{Design of Network Layer}

Design of network layer is core and key to MVTS protocol. It's consisted of three points: one-hop information maintenance, route discovery and data sending. 


\subsection{One-Hop Information Maintenance}

In MVTS protocol, each node needs to maintain the topological information of its own in one-hop range, which is implemented through the maintenance of neighbor information table. In the table, included is nearby node's ID, location information, residual energy and information updating time. It is shown in table1. Where, the state is the "idle", "occupied" and so on.

\section{Table 1. Neighbor Node Information Table}

\begin{tabular}{|l|l|l|l|l|}
\hline $\begin{array}{c}\text { Adjacent } \\
\text { node ID }\end{array}$ & $\begin{array}{c}\text { Coordi } \\
\text { nate }\end{array}$ & $\begin{array}{c}\text { Residua } \\
1 \text { energy }\end{array}$ & $\begin{array}{c}\text { St } \\
\text { ate }\end{array}$ & $\begin{array}{c}\text { Information update } \\
\text { time }\end{array}$ \\
\hline & & & & \\
\hline
\end{tabular}

\subsection{Route Discovery}

Route discovery aims at finding out in the network as many paths from source nodes to convergence nodes as possible. MVTS's route discovery has two mechanisms: greedy forwarding and rollback. Greedy forwarding strategy uses the idea: at each hop, nodes select the best neighboring nodes to resend data. In MVTS, one node i's rating of its adjacent node $\mathrm{j}$ can be got with the expression (1):

$$
f_{i j}=(1-\alpha) \frac{d^{2}(j, D)-d_{\min }^{2}(i)}{d_{\max }^{2}(i)-d_{\min }^{2}(i)}+\alpha \frac{e_{\text {init }}(j)-e_{r e s}(j)}{e_{\text {init }}(j)}
$$

\subsection{Data Sending}

The other task of network layer is to send video data. MVTS sends certain data through not fixed one or several paths, but depending on path situation. That overcomes uneven network energy consumption and helps wholly ensure data transmission quality. Specifically, MVTS used expression (2) to evaluation QoS guarantee level of one path i.

$$
f_{i}=(1-\omega) \frac{h_{i}}{\sum h_{i}}+\omega \frac{n_{i}}{\sum n_{i}}
$$

\section{Experiment Design and Discussion}

\subsection{Testing scene and indicators}

We implemented MVTS solution in NS2 environment and tested on video transmission with myEvalvid [13]. To validate its performance, we compared with AODV, GPSR and TPGF and finished analyses of testing results. Videos for this experiment were chosen from grandma.yuv furnished by Telecommunication Networks Group (TKN) organization.

The parameter setting is shown in Table 2. In each scenario, we calculated the mean value after testing for 10 times.

Table 2. Experimental Parameters Setting

\begin{tabular}{|l|l|}
\hline Parameter & values \\
\hline Scene size & $600 * 400 \mathrm{~m}^{2}$ \\
\hline MAC protocol & 802.11 \\
\hline Node transmission radius & $100 \mathrm{~m}$ \\
\hline Node number & $60,80,100,120,140,160,180,200$ \\
\hline
\end{tabular}




\begin{tabular}{|l|l|}
\hline Ordinary node initial energy & $4 \mathrm{~J}$ \\
\hline Video node initial energy & $30 \mathrm{~J}$ \\
\hline Each frame image size & $176 * 144$ \\
\hline Video stream size & $2000 \mathrm{Frame}$ \\
\hline Video stream transmission speed & $25 \mathrm{Frame} / \mathrm{s}$ \\
\hline
\end{tabular}

We compared four approaches from the following aspects:

(1) Network life cycle: the time when the first node dies in the network; it measures the energy consumption level of protocol;

(2) Packet loss rate: the percentage of total packets reaching convergence nodes to those sent by source nodes;

(3) Frame loss rate: the percentage of image frames reaching convergence nodes to those sent by source nodes; we compared only the rate at I frame;

(4) PSNR: peak signal to noise ratio: a parameter indicator for image quality;

(5) Time delay of frame: the average delay of all frames arriving at sink nodes.

\subsection{Simulation Results and Analyses}

\subsubsection{Network Life Cycle}

Testing results of network life cycle are presented in Fig. 1. From that picture, we see multi-path protocols live longer than single-path ones because after the multi-path protocols are used, node forwarding load becomes balanced and that energy of single node won't be used up too early. TPGF and MVTS used multi-path routing. Compared with TPGF, transmission load of each path of MVTS is more even and thus its work cycle extends.

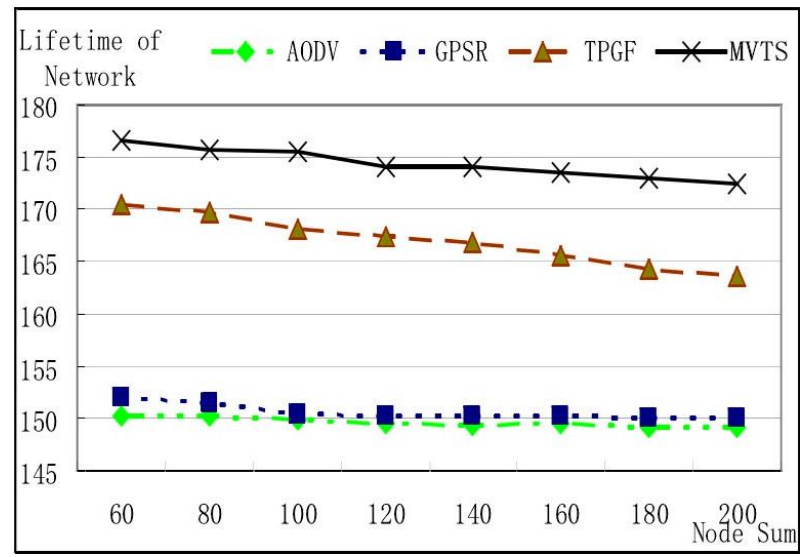

Figure 1. Network Life Cycle Comparison

\subsubsection{Packet Loss Rate}

Testing results of packet loss rate are put in Fig. 2. In it, when nodes are of low density, the packet loss rate of four protocols is basically close. However with increasing nodes, the rate of multi-path protocols descends obviously. The reason is when network size is big, multi-path protocols take advantage of more paths to transmit data, equalizing node burden with ensured transmission reliability. MVTS realizes the lowest packet loss rate owing to the best balance of burdens between each path. 


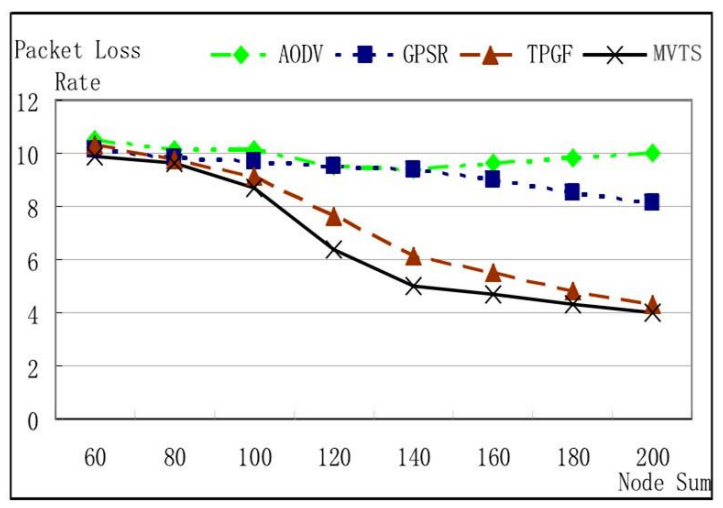

Figure 2. Packet Loss Rate Comparison

\subsubsection{Frame Loss Rate at I Frame}

See Figure 3 about frame loss rate of the four approaches. Similar with packet loss rate, multi-path protocols realizes lower frame loss rate than single-path protocols. In multipath protocols, I frame affects decoding a lot. So MVTS secures especially I frame, at which the packet loss rate is the lowest as indicated there.

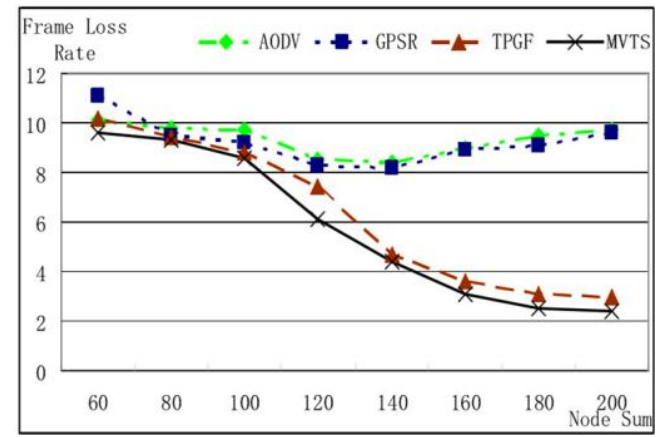

Figure 3. Frame Loss Rate Comparison

\subsubsection{PSNR}

PSNR results are seen in Figure 4. In the figure, multi-path protocols have lower frame loss rate; so the decoding is of higher quality and PSNR value is higher too. As far as MVTS is concerned, it ensures preferentially the transmission of I frame. Hence in all scenes, it gets the highest PSNR value.

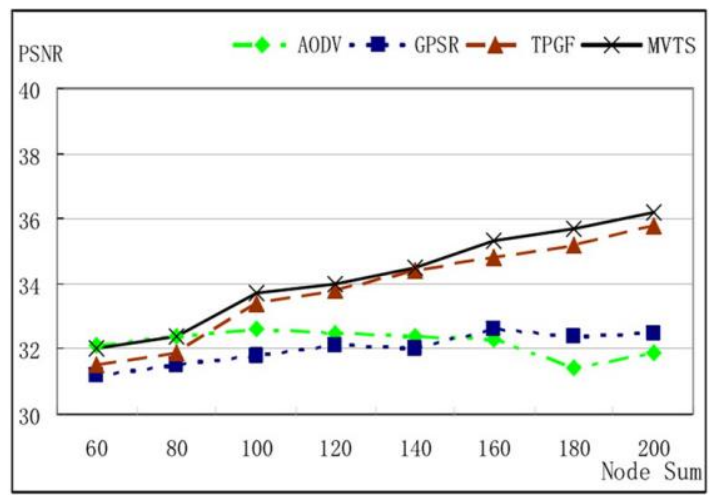

Figure 4. PSNR Comparison 


\subsubsection{Time Delay of Frame}

Figure 5 gives testing results of frame time delay. As found, MVTS's time delay is lower. When video files are of huge data volume, the use of single-path protocol will prolong the queuing time delay of each node on the path. Comparatively, MVTS adopts multi-path transmission mechanism; accordingly, the queuing time delay of each node is shortened and frame's end-to-end time delay becomes shorter.

\subsubsection{Image Transmission Quality}

With regards to image transmission quality, we chose from video data the five consecutive frames, from the 680th to 684th frame, for comparative tests. It is shown in Figure 6. Figure 6(a) shows the image at the 680th frame, the rest in the same manner. Each group of image is composed of five separate images.

For each group of image, in the upper row from the left to right is original image, receiving-end image of respectively AODV and GPSR; in the lower row at the left is transmission result of TPGF and at the right is that of MVTS.

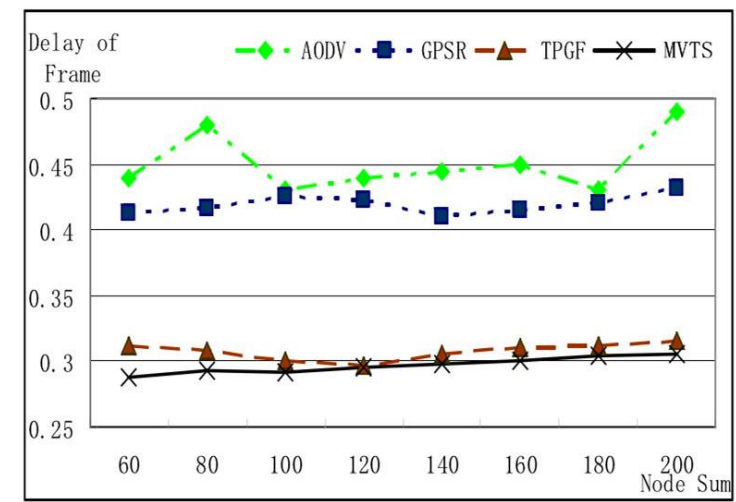

Figure 5. Time Delay of Frame Comparison
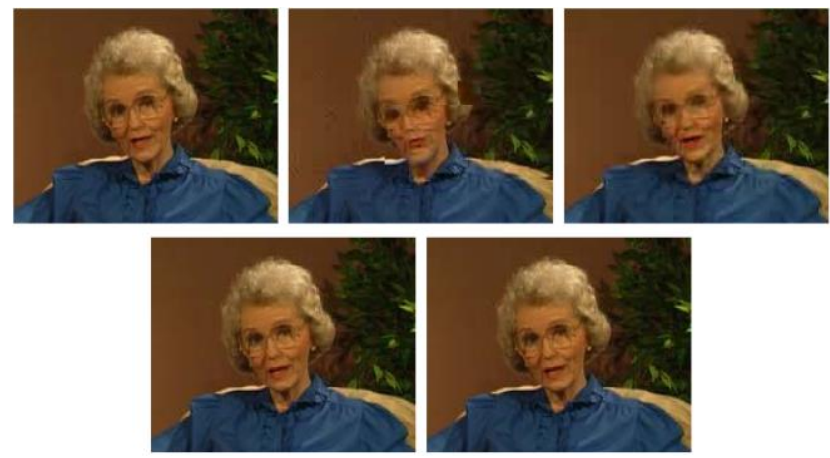

(a) $680^{\text {th }}$ Frame
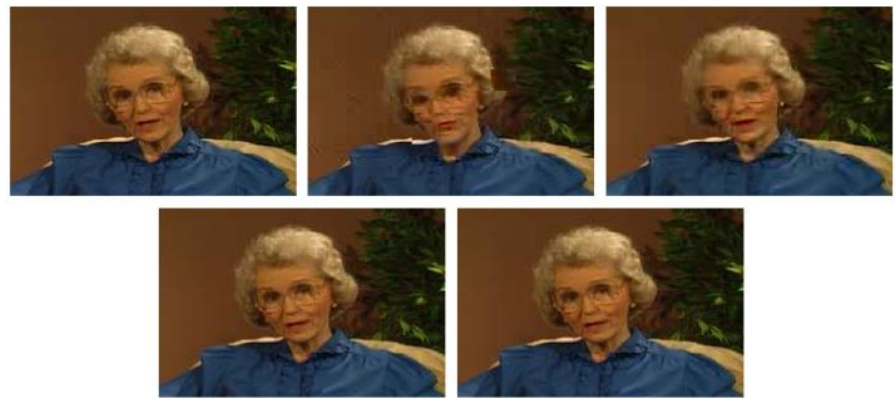
(b) $681^{\text {th }}$ Frame
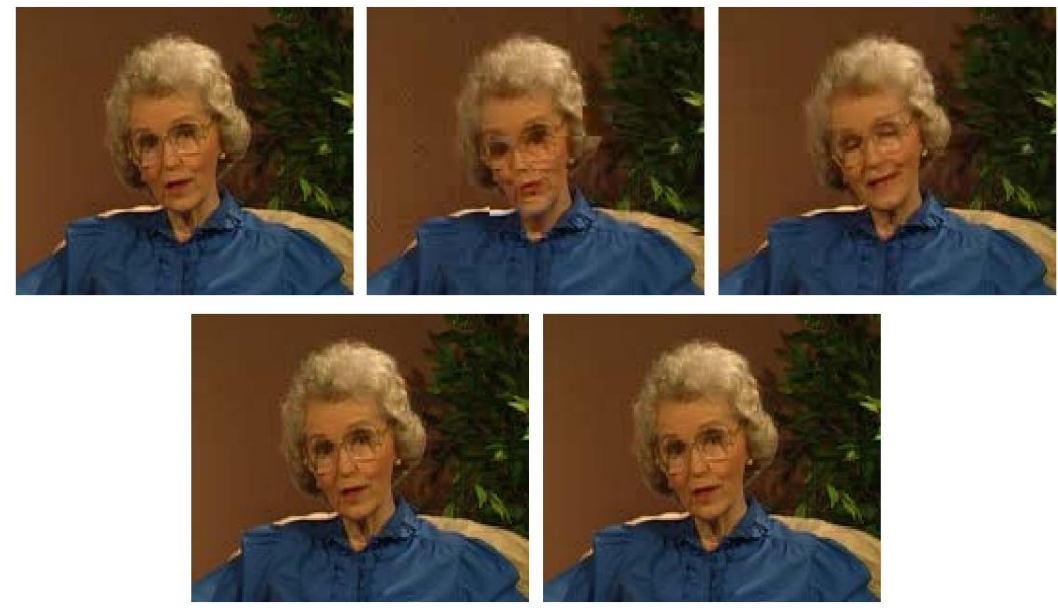

(c) $682^{\text {th }}$ frame
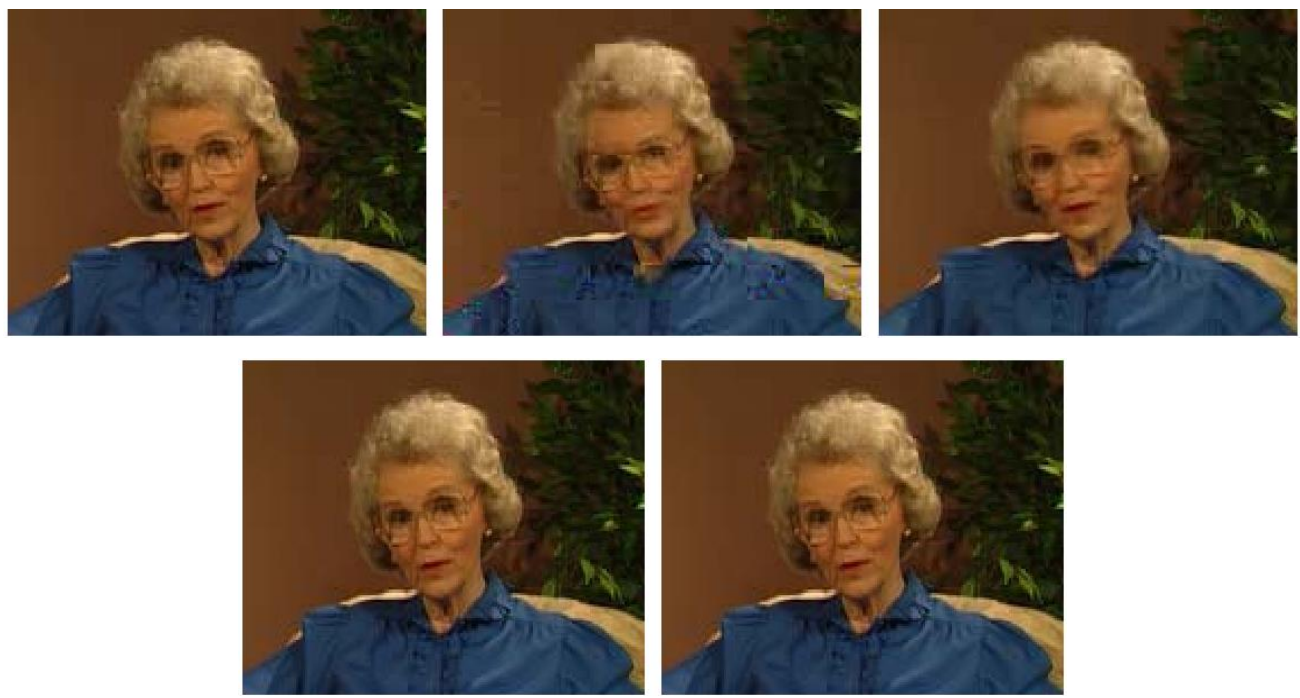

(d) $683^{\text {th }}$ frame
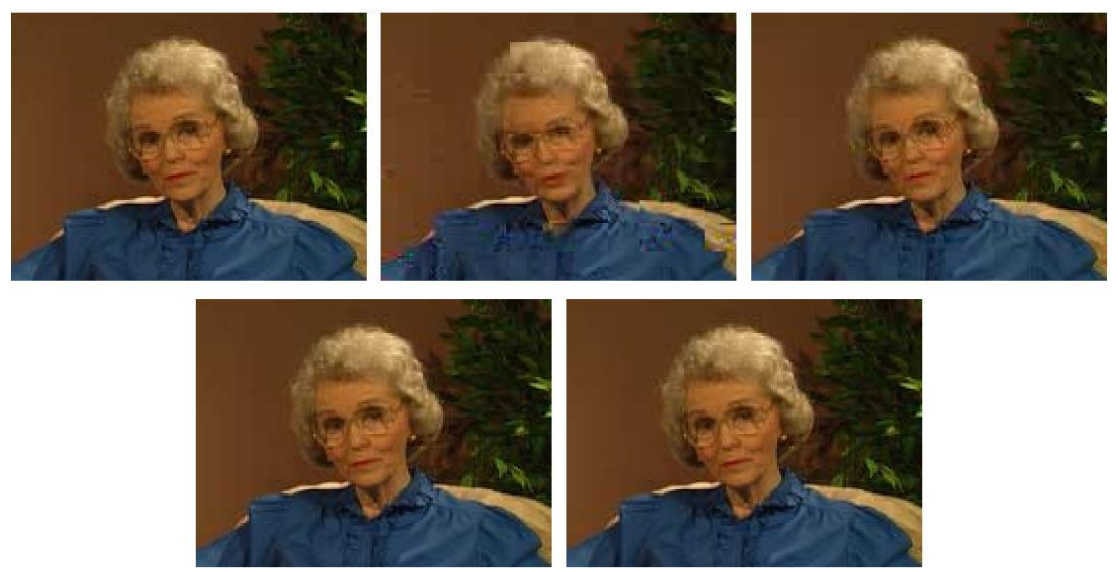

(e) $684^{\text {th }}$ frame

Figure 6. Image Quality Comparison 
Visually from Figure 6, single path routing AODV and GPSR had poor quality of transmission, distorted seriously from original images. In multi-path routing, MVTS and TPGF realized the transmission effect much closer to the original image, no great differences. In terms of network life cycle, MVTS performed much better and that it's more suitable to transmit video data in wireless sensor network.

\section{Conclusion}

In the paper we probed into the transmission quality assurance issue of collected data and designed one cross-layer approach MVTS to address video data collection problem. MVTS introduced differentiated service and multi-path transmission. On the one hand, network load is equalized and network life cycle is extended; on the other hand, data frames in video streams are treated differentially, improving video transmission quality. Experimental results revealed that MVTS is apparently advantageous over existing transmission solutions on the part of video transmission.

\section{References}

[1] M. Xianhu, "Optimization of the throughput of wireless Mesh networks. Fujian Normal University", (2014).

[2] C. Yuanlong, "Research on key technology of the transmission control protocol for intelligent service", Beijing University of Posts and Telecommunications, (2014).

[3] L. Rong, "Research on key technologies in cognitive radio networks", Beijing University of Posts and Telecommunications, (2012).

[4] M. Yalei, "Multipath routing strategy based on network coding", Electronic design engineering, vol. 23, (2013), pp. 50-52.

[5] Jinyong and G.W. Bai, Jinyi, "Based on QoS and distance aware collaborative adaptive error control mechanism", Journal of Beijing University of Posts and telecommunications, vol. 02, (2013), pp. 102106.

[6] L. Hongbing, Q. Xiong, W. Xiaogang, H. Dong and L. Rui, "Progress of layer network fault tolerance technology research in wireless sensor networks", Computer application research, vol. 07, (2013), pp. 1921-1928.

[7] Z. Xiaojuan, L. Yang, S.W. Qiu and Junming, "Wireless sensor network data transmission reliability research", Computer science, vol. 9, (2013), pp. 1-7.

[8] J. Zuqin, H. Jinjin and Ieyasu, "Based on multipath routing secure message transmission protocol modsmt design", Computer applications and software, vol. 9, (2013), pp. 74-76.

[9] L. Lian, S. Limin, and F. Xiaozhong, "A cross layer transmission protocol based on receiver in mobile sensor networks", Computer research and development, vol. 1, (2009), pp. 120-128.

[10] L. Wen, W. Wenbo, J. Xiaojun and Z. Xiangquan, "Analysis of the impact of active route selection on the throughput of multipath parallel transmission", Journal of Beijing University of Posts and Telecommunications, vol. 6, (2014), pp. 54-58.

[11] Z. Xuefei, Z. Guoan and Y. VANET, "A quarter of the cooperative communication cross layer routing design based on television technology", vol. 15, (2014), pp. 140-143.

[12] Z. Wei, L.W. Min, L. Guangye and Yunchong, "Based on application layer traffic optimization of the relay path selection scheme", Computer engineering and science, vol. 10, (2014), pp. 1880-1887.

[13] C.H. Ke, C.K. Shieh and W.S. Hwang, "An Evaluation Framework for More Realistic Simulations of MPEG Video Transmission”, Journal of Information Science and Engineering, vol. 24, no. 2, (2008), pp. 425-440.

\section{Author}

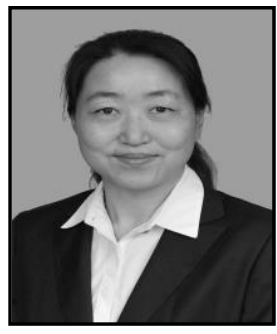

Guo WeiWei, She received her B.S degree in computer Science from Heilongjiang University of Science and Technology. She received her M.S degree in computer Science from Liaoning Technical University. She is an Associate professor in Heilongjiang University of Technology. Her research interests include database and algorithm. 
International Journal of Future Generation Communication and Networking Vol. 9, No. 2 (2016) 